\title{
Effects of Mobile Business Processes on the Software Process
}

\author{
André Köhler, Volker Gruhn \\ University of Leipzig \\ Chair of Applied Telematics / e-Business \\ Klostergasse 3 \\ 04109 Leipzig, Germany \\ \{koehler,gruhn\}@ebus.informatik.uni-leipzig.de
}

\begin{abstract}
The adoption of mobile technologies into companies frequently follows a technology-driven approach without precise knowledge about the potential benefits that may be realised. Especially in larger organisations with complex business processes, a systematic procedure is required if a verifiable economic benefit is to be created by the use of mobile technologies. Therefore, the term "mobile business process" is defined in this paper. Subsequently, we introduce a procedure for the systematical analysis of the distributed structure of a business process model in order to identify requirements for software eng $i$ neering in mobile sub-processes. For that purpose, the method Mobile Process Landscaping is used to decompose a process model into different levels of detail. The method aims to manage the complexity and limit the process analysis to the potentially mobile sub-processes from the beginning. The result of the analysis can be used on the one hand as a foundation for the redesign of the business processes and on the other hand for the requirements engineering of mobile information systems.
\end{abstract}

\section{Motivation}

The orientation towards business processes and their optimisation has been an important issue for some years [1]. The identification of opportunities for cost reduction, as well as integrated IT-support for processes are increasingly coming to the fore, with technologies supporting mobility making an important contribution. Particular potential benefits lie in the seamless, companycomprehensive integration of all partners participating in the business process, especially when this process is distributed [2]. Thus, each process-step on the value chain can be connected directly to the operational information processing, so the goal-oriented control of the whole business process becomes feasible [3].

To exploit these advantages effectively, specialized information systems, supporting not only the business process but handling the mobility of the process-executing person if necessary, are required. In order to develop such systems we propose the method Mobile Process Landscaping. This method allows the goal-oriented analysis of a process model and its distribution structure to explore mobilisation opportunities, as well as deducing requirements for the software engineering process.

\section{Mobility within Business Processes and In- formation Systems}

In the following, only business processes with a specific distribution structure and thus a certain mobility of the process-executing persons are considered. We propose that mobility is given when for at least one process partition

a) there is an "uncertainty of location",

b) this "uncertainty of location" is externally determined, and

c) a cooperation with external resources (from the process-point of view) is needed in the execution of the process.

The assumption a) is based on the concept of "location uncertainty" by Valiente and van der Heijden [4], according to which the place of the execution of an activity can be different in different instances of the business process or the places can change during the execution of an activity. Thus, we deal with a mobile process partition within a business process. Because multiple mobile process partitions are conceivable, and a mobile process partition often affects the whole business process, the complete business process is called "mobile business process".

Further on, assumption b) presumes that the location uncertainty is caused by external factors and that the process-executing person has therefore no freedom of choice regarding the place of the process execution. Assumption c) restricts the term „mobile business process“ to the necessity of cooperation with external esources within the considered process partition, for instance caused by the need for communication or coordination with other persons or interaction with other objects.

We see the necessity of representing the workflow in a company by a specialised process model in order to identify potential process improvements on the basis of the definition of mobile business processes. Subsequently, the requirements for the creation of the mobile element of the 
existing information system need to be defined in order to be able to examine whether the mobile technology and devices meet the demands. For this procedure, we propose the following steps [5]:

(1) Analysis of the process model and identification of mobile business processes.

(2) Redesign of the identified process partitions (under the assumption of the producibility of a mobile element for the information system).

(3) Specification of the mobile element as required by the new business processes.

(4) Validation of the profitability of the change (valuation of the relationship between estimated costs and forecast benefit).

(5) Implementation of the change (actual redesign of the processes and development of the mobile element).

In the following, we briefly describe the proposed procedure.

\section{Requirements for Mobile Information Sys- tems}

\subsection{Identification of Mobile Business Processes}

With Mobile Process Landscaping, we propose a systematical approach for identifying and analysing mobile business processes. It is a specialisation of the "Process Landscaping" method [6]. The idea of the method is to split the modelling of processes into different tiers, starting with a coarse and simplified form of the process description and then increasing the level of detail with each tier. Other approaches for the modelling of (mobile) processes (see e.g. [7], [4], [8]) neglect the question of the level of detail.

The aim of the proposed procedure is twofold: on one hand, it should help to handle the complexity of processes. On the other hand, it should help to recognise the distribution structure very early. Thus, the method can only be applied to distributed process structures. At an early stage, process partitions with mobile potentials can be located. The process analysis should just at these points be continued in order to minimise the analysis effort. For the presentation of the different levels of detail, we propose four different tiers, that are determined by the global company structure.

Figure 1 shows the correlation between the levels of detail in the process model and the real world according to [9]. Applying this procedure in a top-down-approach to process modelling, we identify the process partitions which need to be redesigned in order to be supported by a mobile information system. Our aim is the identification of mobile potentials on each level of detail. A refinement of the process model on the next level of detail is just needed for identified parts. This way, the complexity can be reduced and the effort for the analysis can be minimised.

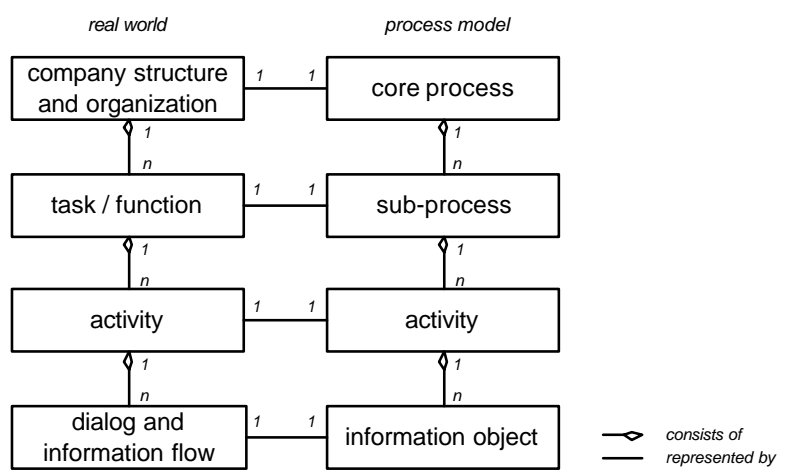

\section{Fig. 1. Correlation between the levels of detail in the process model and the real world}

The lowest level of the resulting process model is the dialog and information flow in the real world consisting of single activities. Transmitted into the process model it is represented by information objects which are interchangeable within spatially separated parts of an organis ation. The modelling of this lowest level is crucial for describing the demands on an information system which is supposed to support the process model. Based on the model one can deduce concrete requirements for the software process.

Figure 2 clarifies this procedure using an example from the insurance industry. Organisational units whose subjects (single persons or groups of persons) are not spatially separated from each other are symbolized by a grey rectangle with rounded corners. Processes inside organis ational units are represented by a white rectangle. Relations between processes inside an organisational unit are symbolized by a solid line (internal interaction). A dashed line indicates a relation between processes in different organisational units (external interactions).

The aim of this kind of description is to identify mobile potentials within the considered processes which, according to our definition, is induced by an externally determinated location uncertainty as well as a cooperation with external resources.

An externally determinated location uncertainty is present in processes that cannot be allocated clearly to a single organisational unit. This means, multiple, spatially separated persons or groups of persons are involved in their execution. This is a necessary, but not sufficient condition for a mobile business process. If such a partition is identified in the process model, we assume a potential mobility that can be approved or disproved by a stepwise refinement of the process partition. The potential mobile process partition is indicated in the graphical representation by different processes lying on top of each other. The 
cooperation with external resources is shown by a dashed line, indicating an exchange of information between different process partitions across organisational units. This is also a necessary, but not sufficient condition for a mobile business process. Again, the potential mobility can be approved or disproved by a stepwise refinement of the process partition.

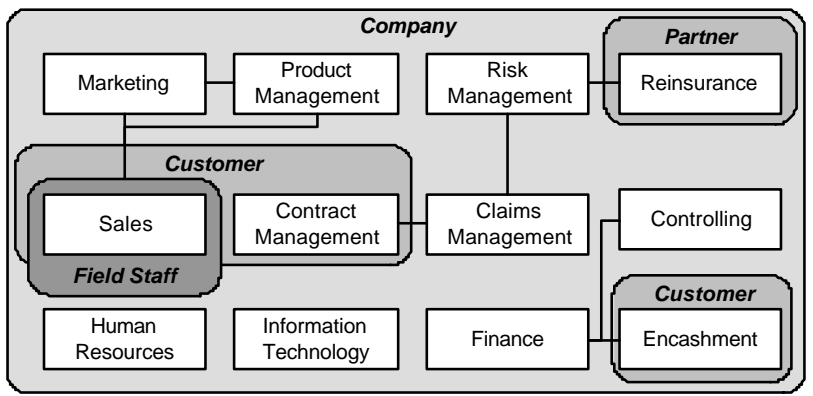

Fig. 2. Core Processes in the insurance industry

In this case the company itself, the customers, the field staff and the partners represent organisationally separated units. Involving three spatially separated units, the sales process is potentially mobile.

The further analysis of the sales process should adequately comprise the design and the analysis of the subprocesses and the single activities. At this point we forbear from exemplifying the according procedure. Figure 3 shows a small section from the lowest level of the process model in which the information objects are modelled.

The field staff prints a data sheet for submitting the customer data and sends it to the company via mail. There, the data is again acquired and saved. Among the information objects "send mail" and "acquire data", an information exchange across the boundaries of spatially separated organisational units takes place, so both information objects are potentially mobile.

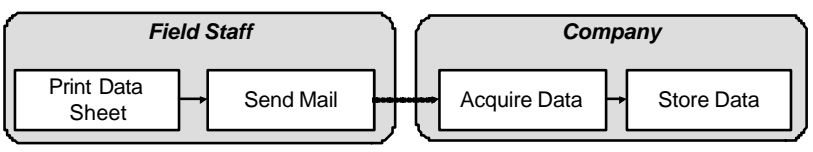

\section{Fig. 3. Information Objects in the Sales Process}

The information objects "send mail" and "acquire data" highlight a process partition which renders the activity ,transfer customer data“, the sub process ,tender preparation“ and the core process ,sales“ potentially mobile. If we execute the analysis for each process partition identified as potentially mobile, more mobile parts would probably be identified.

At this point, a redesign of the process can be applied in order to develop a mobile information system, as proposed in step (2) of our procedure in chapter 2. All processes identified as potentially mobile as well as their preliminary and subsequent elements, come into question for a redesign. In our example, those processes are „send mail“" and ,,acquire data“, as well as the preliminary process ,print data sheet“ and the subsequent process ,store data". In this case, the whole process partition is affected. It should be considered that preliminary and subsequent elements on a superior level of detail could be affected, too. Concerning those, an analysis up to the fourth level of detail needs to be conducted in order to identify the specific preliminary and subsequent information objects.

\subsection{Redesign of the Identified Process Partitions}

The next step towards modelling a mobile information system contains the redesign of the processes which were identified as being mobile.

This implies, that the considered processes are designed under the assumption that mobile technologies enable the connection to an existing information system or one that yet has to be created. However, at this point the actual availability of concrete mobile technologies has to be entirely ignored.

A redesign of the process exemplified in figure 3 may imply that customer data should be conveyed electronically to the company. As a result the objects "printing the data sheet", "sending the mail" and "acquiring the data in the company" become redundant. Considering that the field staff transfers the data, which is already acquired in the software, from the notebook to the company by mouse click, the revised process is noticeably shorter. The company in turn is able to store the customer data directly in the transferred format. Figure 4 shows the result of the redesign.

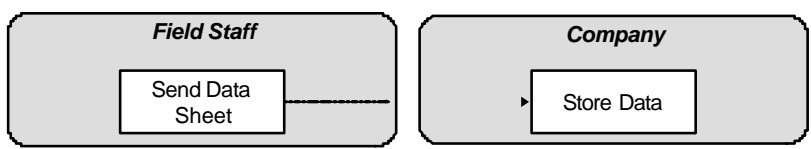

\section{Fig. 4. Redesigned Part of the Sales Process}

\subsection{Specification of the Mobile Element}

The result of the redesigned mobile process part has to be supported by an adequate information system. The requirements on the mobile part of the information system can be derived directly from the modified process. In our example (see Fig. 4), the opportunity to convey the $\mathbf{x}$ quired customer data electronically to the company has to be provided to the field staff. Within the agency they may use the company network, en-route mobile technologies might be suitable.

Additionally the company has to create an interface which facilitates the electronical acquisition and storage of the customer data conveyed by the field staff. In this step concrete requirements on the future software development are defined. 


\subsection{Validation of the Profitability of the Change}

A very important part of the proposed procedure is represented by the validation of the profitability of the change [5]. It concerns an appraisal if the desired process change and the costs involved are justified by the evolving benefit.

The expense for such a change basically results from the reorganisation of processes, the software development and the purchase of mobile devices. On the other hand, there is a change in the costs created by the execution of a process. For example, certain activities are dropped out or replaced by newly created and more resource-efficient ones. A precise estimation of the resulting process cost can be deduced from a simulation of the process model.

\subsection{Implementation of the Change}

If the planned change is considered as economic reasonable one can start with the precise implementation of the changed process. During this step it is necessary to develop the software, to identify suitable mobile devices, to conduct a usability evaluation of the final solution and to train the field staff.

\section{Conclusion}

We proposed a procedure for a systematical analysis of mobile potentials in business processes. The first step of this procedure was conducted by using the Mobile Process Landscaping method. At this point, we see a need for further research in order to integrate the shown procedure into the established approaches for modeling business processes. Finally, the drafted procedure aims to support the development of mobile information systems whose characteristics are not defined by mobile technology, but are rather deduced from the requirements of newly designed mobile business processes.

\section{Acknowledgements}

The Chair of Applied Telematics / e-Business is endowed by Deutsche Telekom AG.

\section{References}

[1] A. Scheer, Business process engineering: reference models for industrial enterprises. Tokyo: Sp ringer, 1998.

[2] V. Gruhn and U. Wellen, "Software Support for Distributed Business Processes," in Proceedings of the Sixth Asia Pacific Software Engineering Conference, IEEE Computer Society Press, 1999, pp. 200-206.

[3] C. W. Craighead and N. G. Shaw, "E-commerce value creation and destruction: a resource-based, supply chain perspective," ACM SIGMIS Database. vol. 34, pp. 39-49, 22003.

[4] P. Valiente and H. van der Heijden, A method to identify opportunities for mobile business processes. 2002.

[5] A. Köhler and V. Gruhn, "Mobile Process Landscaping am Beispiel von Vertriebsprozessen in der Assekuranz," in Mobile Economy - Transaktionen, Prozesse, Anwendungen und Dienste, vol. P-25, K. Pousttchi and K. Turowski, Ed. Bonn: Köllen Druck + Verlag GmbH, 2003, pp. 12-24.

[6] V. Gruhn and U. Wellen, "Process Landscaping: Modeling Distributed Processes and Proving Properties of Distributed Process Models," vol. 2128, Springer, 2001, pp. 103-125.

[7] N. M. M. Noor, K. N. Papamichail and B. Warboys, "Process Modeling for Online Communications in Tendering Processes," in Proceedings of the 29th EUROMICRO Conference 'New Waves in System Architecture', IEEE Computer Society, 2003, pp. 17-24.

[8] T. Ritz and M. Stender, "Modeling of B2B Mobile Commerce Processes," in 17th International Conference on Production Research ICPR-17, Blacksburg: Virginia Tech, 2003,.

[9] V. Gruhn and U. Wellen, "Structuring Complex Software Processes by 'Process Landscaping'," in EWSPT European Workshop on Software Process Technology, Kaprun, Austria, vol. 1780, Springer, 2000, pp. 138-149. 